Research

Open Access

\title{
Optimizing intensive care capacity using individual length-of-stay prediction models
}

\author{
Mark Van Houdenhoven ${ }^{1,4,5}$, Duy-Tien Nguyen ${ }^{1,2}$, Marinus J Eijkemans ${ }^{2}$, Ewout W Steyerberg', \\ Hugo W Tilanus ${ }^{3}$, Diederik Gommers ${ }^{4,5}$, Gerhard Wullink ${ }^{1,4,5}$, Jan Bakker ${ }^{5}$ and Geert Kazemier ${ }^{1,3}$
}

\begin{abstract}
1Department of Operating Rooms, Erasmus University Medical Center, P.O. Box 2040, 3000 CA Rotterdam, The Netherlands 2Department of Public Health, Erasmus University Medical Center, P.O. Box 2040, 3000 CA Rotterdam, The Netherlands ${ }^{3}$ Department of Surgery, Erasmus University Medical Center, P.O. Box 2040, 3000 CA Rotterdam, The Netherlands ${ }^{4}$ Department of Anesthesiology, Erasmus University Medical Center, P.O. Box 2040, 3000 CA Rotterdam, The Netherlands ${ }^{5}$ Department of Intensive Care, Erasmus University Medical Center, P.O. Box 2040, 3000 CA Rotterdam, The Netherlands

Corresponding author: Gerhard Wullink, g.wullink@erasmusmc.nl
\end{abstract}

Received: 7 Dec 2006 Revisions requested: 14 Jan 2007 Revisions received: 16 Feb 2007 Accepted: 27 Mar 2007 Published: 27 Mar 2007

Critical Care 2007, 11:R42 (doi:10.1186/cc5730)

This article is online at: http://ccforum.com/content/11/2/R42

(C) 2007 Van Houdenhoven et al.; licensee BioMed Central Ltd.

This is an open access article distributed under the terms of the Creative Commons Attribution License (http://creativecommons.org/licenses/by/2.0), which permits unrestricted use, distribution, and reproduction in any medium, provided the original work is properly cited.

\begin{abstract}
Introduction Effective planning of elective surgical procedures requiring postoperative intensive care is important in preventing cancellations and empty intensive care unit (ICU) beds. To improve planning, we constructed, validated and tested three models designed to predict length of stay (LOS) in the ICU in individual patients.

Methods Retrospective data were collected from 518 consecutive patients who underwent oesophagectomy with reconstruction for carcinoma between January 1997 and April 2005. Three multivariable linear regression models for LOS, namely preoperative, postoperative and intra-ICU, were constructed using these data. Internal validation was assessed using bootstrap sampling in order to obtain validated estimates of the explained variance $\left(r^{2}\right)$. To determine the potential gain of the best performing model in day-to-day clinical practice, prospective data from a second cohort of 65 consecutive patients undergoing oesophagectomy between May 2005 and April 2006 were used in the model, and the predictive
\end{abstract}

performance of the model was compared with prediction based on mean LOS.

Results The intra-ICU model had an $\mathrm{r}^{2}$ of $45 \%$ after internal validation. Important prognostic variables for LOS included greater patient age, comorbidity, type of surgical approach, intraoperative respiratory minute volume and complications occurring within 72 hours in the ICU. The potential gain of the best model in day-to-day clinical practice was determined relative to mean LOS. Use of the model reduced the deficit number (underestimation) of ICU days by 65 and increased the excess number (overestimation) of ICU days by 23 for the cohort of 65 patients. A conservative analysis conducted in the second, prospective cohort of patients revealed that $7 \%$ more oesophagectomies could have been accommodated, and 15\% of cancelled procedures could have been prevented.

Conclusion Patient characteristics can be used to create models that will help in predicting LOS in the ICU. This will result in more efficient use of ICU beds and fewer cancellations.

\section{Introduction}

Intensive care units (ICUs) consume a considerable portion of hospital budgets. Moreover, costs are predicted to rise with the emergence of new treatment methods. Problems with ICU capacity are nevertheless common, and studies conducted in ICUs have documented high rates of refusal to admit because of lack of empty beds [1,2]. In addition, the need to serve the 'greying' population is likely to increase demand for ICU beds further, exacerbating the current strain on ICU capacity. Con- sequently, hospitals will face an increase in numbers of cancelled surgical procedures that necessitate postoperative intensive care, and higher rates of refusal to admit other critically ill patients $[2,3]$. The only way to remedy these problems is apparently to improve the efficiency with which the available ICU and operating room capacity is used, in other words to optimize patient planning.

$\overline{\mathrm{Cl}}=$ confidence interval; $\mathrm{ICU}=$ intensive care unit; LOS = length of stay. 
Patient planning depends importantly on reliable and adequate management information. Key elements in the ICU setting are the patient's expected length of stay (LOS) in the ICU at admission and possible changes in expected LOS resulting from later treatment. Starting from the admission date and expected LOS, the planner will be able to pinpoint the anticipated date at which an ICU bed will once again become available. This information, along with subsequent changes in a patient's expected LOS, is needed to schedule the next operating room patient who requires postoperative intensive care or to reserve emergency patient capacity on the ICU. In addition, information on expected LOS preoperatively facilitates scheduling of individual surgical procedures on specific dates. This information can be used to predict ICU admission dates and LOSs. Information that emerges during the surgical procedure and the postoperative stay in the ICU can influence the LOS predicted by the preoperative model. This so-called online patient planning can help to improve OR and ICU programmes.

Clinicians generally assume that LOS of individual patients is unpredictable. Intensivists are expected to be able predict LOS roughly, but the accuracy of this prediction depends largely on the intensivist's experience. We speculate that if comprehensive evaluations of the association between preoperative, intraoperative and postoperative prognostic variables on the one hand, and LOS on the other are translated into a mathematical model, then this model might be able to predict LOS with greater accuracy.

The main goal of this study was to develop a model that will provide planners with a tool to predict the LOS of individual patients in the ICU. Data on a cohort of consecutive patients undergoing an elective oesophagectomy were used to create and validate such models. Predictive power was assessed to determine the best performing model. In a second cohort of patients, the LOSs of individual patients were predicted prospectively to determine the potential gain of this best model on a day-to-day basis.

\section{Methods \\ Data}

Data from 518 consecutive patients who underwent elective oesophagectomy with reconstruction for carcinoma at the Erasmus University Medical Centre, Rotterdam, The Netherlands, between January 1997 and April 2005, were retrieved from the hospital information system. These data were combined with detailed data from a prospective database held at the Department of Surgery. The Erasmus University Medical Centre includes a total of 1,212 beds on several locations. It is a trauma centre for a catchment area that includes 5.2 million people. The main site includes 32 ICU beds and 19 operating rooms.
The outcome variable of the present study was LOS, defined as the time in days between admission and discharge from the ICU. Admission to and discharge from the ICU were based on the national protocol [4]. For patients discharged to the ward and readmitted to the ICU within 48 hours, the intervening stay on the ward was included in the LOS. Definitions of these variables and supporting references [5-7] are given in Table 1; reports that provide evidence supporting the use of these variables in the model are also referenced [8-12].

\section{Model construction}

Only those variables that were present in more than $1 \%$ of the patients were included, in order to avoid unstable estimates. On clinical grounds, two expert surgeons (HWT and GK) and two expert anaesthetists (DG and JB) formed a preselection of factors from the potentially prognostic variables in order to prevent overfitting [13-15]. Only these selected variables were used to build the three models. In those patients with missing values, data were completed using multiple imputation methods. This was done under the assumption that the distribution of the missing date and the complete data were the same [16].

The imputed model included both the independent potentially prognostic variables and the outcome variable LOS. Given the inherently skewed distribution of LOS, a natural log transformation was used [17].

Univariate linear regression analysis was used to test which of the variables contributed to LOS with $P \leq 0.20$. The mean and standard deviation are reported for those variables that are normally distributed. The median and interquartile ranges are given for non-normal distributions.

Significant variables in the univariate analyses were entered as potentially prognostic variables into a backward, stepwise selection procedure to construct a multivariable linear model that provides a natural logarithm transformed prediction of LOS (In [LOS]). Because LOS can be predicted based on expanding sets of available information at three stages, three multivariable linear models were constructed. First, preoperative data were used to build a preoperative prediction model. Then, intraoperative data were incorporated to construct a postoperative model. To construct an intra-ICU model, which was used after three days on the ICU, all selected data were used. This final model was constructed to improve accuracy based on new information from the last three ICU days. The criterion for retention of variables in the model was $P<0.20$, which ensured high power for inclusion of variables with somewhat weaker predictive effects [14]. Interactions between variables and nonlinear relationships were explored. A smearing factor to correct the 'back transformation' bias was needed to obtain the estimated LOS, because a natural logarithmic transformation on LOS was used [18]. Goodness-of-fit was assessed graphically by plotting observed LOS against predicted LOS in a calibration plot. The predictive power of the 
Table 1

Characteristics for both cohorts of patients who underwent oesophagectomy with reconstruction for cancer

\begin{tabular}{|c|c|c|c|}
\hline & Construction sample $(n=518)$ & Application sample $(n=65)$ & Reference \\
\hline \multicolumn{4}{|l|}{ Patient characteristics } \\
\hline Age (years) & $63(55-70)$ & $60(56-68)$ & [8-12] \\
\hline Male sex & $407(79)$ & $48(74)$ & [9-12] \\
\hline $\mathrm{BMI}\left(\mathrm{kg} / \mathrm{m}^{2}\right)$ & $25(22-28)$ & $26(23-29)$ & [8] \\
\hline ASA 1,2 & $89(17)$ & $28(43)$ & [5] \\
\hline Hypertension & $192(37)$ & $35(54)$ & [6] \\
\hline Previous stomach operation & $132(25)$ & $19(29)$ & [6] \\
\hline Preoperative serum haemoglobin (mmol Fe/l) & $8.4(7.6-9.2)$ & $8.7(7.4-9.4)$ & {$[11,12]$} \\
\hline Preoperative serum creatinin $(\mu \mathrm{mol} / \mathrm{l})$ & $78(68-89)$ & $78(68-90)$ & {$[11,12]$} \\
\hline Preoperative $\mathrm{FEV}_{1}(\mathrm{I})$ & $2.9(2.4-3.5)$ & $3.2(2.4-3.7)$ & \\
\hline Preoperative chemotherapy & $170(33)$ & $17(26)$ & {$[8,10]$} \\
\hline Preoperative radiotherapy & $55(11)$ & $8(12)$ & {$[8,10]$} \\
\hline \multicolumn{4}{|l|}{ Aetiology } \\
\hline Gastroesophageal reflux disease & $63(12)$ & $9(14)$ & [6] \\
\hline Barrett's esophagus & $43(8)$ & $9(14)$ & [6] \\
\hline Other & $66(13)$ & $13(20)$ & [6] \\
\hline \multicolumn{4}{|l|}{ Comorbidities } \\
\hline Cardiac & $134(26)$ & $24(37)$ & [6] \\
\hline Respiratory & $91(17)$ & $7(11)$ & [6] \\
\hline Vascular & $65(13)$ & $6(9)$ & [6] \\
\hline Neurological & $33(6)$ & $7(11)$ & [6] \\
\hline Diabetes mellitus & $51(10)$ & $7(11)$ & [6] \\
\hline Other carcinoma & $53(10)$ & $4(6)$ & [6] \\
\hline Other & $40(8)$ & 2 (3) & [6] \\
\hline \multicolumn{4}{|l|}{ Tumour characteristic } \\
\hline Adenocarcinoma & $340(66)$ & $51(79)$ & \\
\hline pTNM stage 0 & $27(5)$ & $7(11)$ & [7] \\
\hline I & $64(12)$ & $5(8)$ & [7] \\
\hline lla & $120(23)$ & $13(20)$ & [7] \\
\hline $\mathrm{llb}$ & $46(9)$ & $5(8)$ & [7] \\
\hline III & $193(37)$ & $21(32)$ & [7] \\
\hline IV & $68(13)$ & $14(22)$ & [7] \\
\hline Radicality (R0) & $400(77)$ & $54(83)$ & \\
\hline
\end{tabular}


Critical Care Vol 11 No 2 Van Houdenhoven et al.

Table 1 (Continued)

Characteristics for both cohorts of patients who underwent oesophagectomy with reconstruction for cancer

Session variables

\begin{tabular}{|c|c|c|c|}
\hline Expected duration of the procedure (min) & $240(180-270)$ & $266(262-314)$ & \\
\hline Duration of the procedure (min) & $301(254-359)$ & $333(290-368)$ & [11] \\
\hline Total age of the two head surgeons (years) & $83(72-88)$ & $84(74-94)$ & \\
\hline Transthoracic approach & $114(22)$ & $14(22)$ & {$[8,10-12]$} \\
\hline Reconstruction using colon & $24(5)$ & $3(5)$ & {$[10-12]$} \\
\hline Oesophagus and cardia resection & $506(98)$ & $65(100)$ & [6] \\
\hline Splenectomy during surgical procedure & $15(3)$ & $2(3)$ & \\
\hline Absolute crystalloid administration (I) & $6.0(4.5-7.0)$ & $4.0(2.3-5.5)$ & [8] \\
\hline Absolute colloid administration (I) & $1.5(1.5-2.0)$ & $1.5(1.5-2.0)$ & {$[8]$} \\
\hline Erythrocyte concentrate transfusion & $276(53)$ & $22(33)$ & {$[8,12]$} \\
\hline Fresh frozen plasma transfusion & $36(7)$ & $6(10)$ & {$[8,12]$} \\
\hline Absolute blood loss (I) & $1.5(1.0-2.2)$ & $1.1(0.7-1.5)$ & {$[8,12]$} \\
\hline Absolute urine production (I) & $0.7(0.4-1.3)$ & $0.4(0.3-0.7)$ & \\
\hline Epidural analgesia during procedure & $467(90)$ & $57(88)$ & \\
\hline Vasopressor administration & $214(41)$ & $63(97)$ & \\
\hline Duration of vasopressor therapy (hours) & $0(0-1.5)$ & $270(206-337)$ & \\
\hline Minute volume (I) & $7.8(7.2-8.8)$ & $7.8(7.0-8.4)$ & \\
\hline Positive end-expiratory pressure $\left(\mathrm{cmH}_{2} \mathrm{O}\right)$ & $5(4-7)$ & $6(5-7)$ & \\
\hline Serum oxygen saturation (\%) & $98(96-100)$ & $100(98-100)$ & \\
\hline End temperature $\left({ }^{\circ} \mathrm{C}\right)$ & $35.8(35.2-36.4)$ & $36.3(36.0-36.9)$ & \\
\hline Lactate $(\mathrm{mmol} / \mathrm{l})$ & $1.7(1.2-2.2)$ & $1.4(1.0-2.0)$ & \\
\hline \multicolumn{4}{|l|}{ Postoperative variables } \\
\hline Duration of mechanical ventilation & $0.63(0.13-5.58)$ & $0.54(0.12-4.12)$ & \\
\hline \multicolumn{4}{|l|}{ Surgical complications } \\
\hline Postoperative bleeding & $19(4)$ & $1(2)$ & {$[6]$} \\
\hline Chylothorax & $20(4)$ & $3(5)$ & {$[6]$} \\
\hline Leakage of anastomosis & $38(7)$ & $9(13)$ & [6] \\
\hline Necrosis of anastomosis & $18(4)$ & $2(3)$ & {$[6]$} \\
\hline Other & $42(8)$ & $11(17)$ & {$[6]$} \\
\hline \multicolumn{4}{|l|}{ Nonsurgical complications } \\
\hline Pulmonary: pneumonia, atelectasis, or ARDS & $198(38)$ & $24(37)$ & {$[6]$} \\
\hline Infection: urinary tract, sepsis & $31(6)$ & $1(2)$ & {$[6]$} \\
\hline Thrombosis, embolism & $20(4)$ & $3(5)$ & {$[6]$} \\
\hline Other & $135(26)$ & $26(40)$ & {$[6]$} \\
\hline Length of stay in the ICU (days) & $4.0(2.0-7.9)$ & $4.2(2.9-7.9)$ & \\
\hline
\end{tabular}

Values are expressed as number (\%) or, for continuous variables, as median (25th to 75th percentile). ARDS, acute respiratory distress syndrome; ASA, American Society of Anaesthesiology Physical Status Score; BMI, body mass index; FEV1, forced expiratory volume in 1 s; ICU, intensive care unit. 
Figure 1

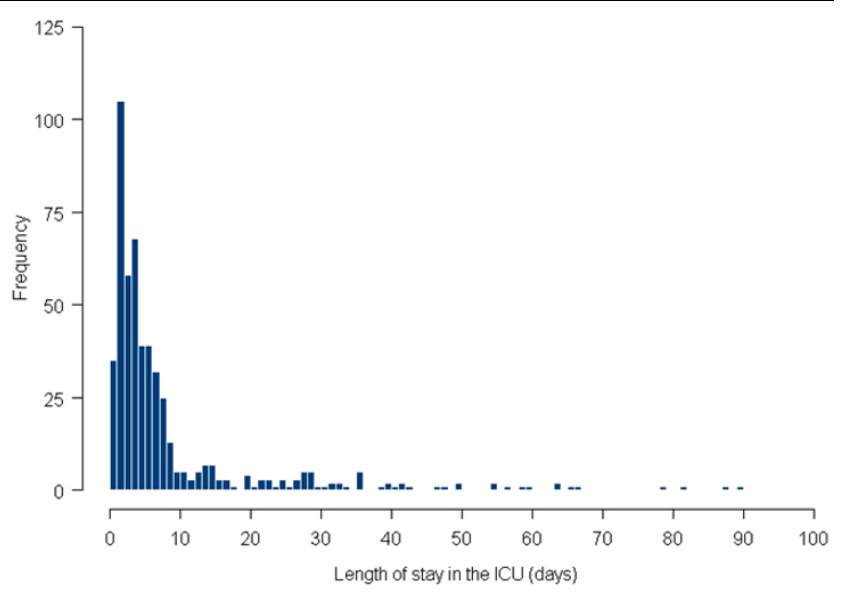

Distribution of length of stay in the ICU. ICU, intensive care unit.

model was expressed as the percentage explained variation (multiple $\mathrm{r}^{2}$ ) on the logarithmic scale.

Internal validity was assessed with bootstrap sampling to obtain estimates of the optimism of the multiple $r^{2}[14,19,20]$. This optimism indicates the expected decrease in model performance when it is applied in future patients [21]. Bootstrap samples were drawn with replacement and with the same size as the original sample. Regression models were constructed in each bootstrap sample and tested on the original sample. This was repeated 200 times to obtain stable estimates of the optimism of the model [21].

Analyses were performed using SPSS version 11 (SPSS Inc, Chicago, IL, USA) and S-Plus version 6 (Insightful Inc, Seattle, WA, USA).

\section{Model application}

After internal validation of the models, the gain in terms of usage of ICU capacity with the model exhibiting the highest $r^{2}$ was assessed in routine clinical practice. Prospective data were collected for consecutive patients who underwent elective oesophagectomy with reconstruction for carcinoma. The data were collected during the period from May 2005 to April 2006, which were the 12 months after construction of the model (Table 1). The prediction model was assessed by comparing the total overestimation and underestimation of the required ICU days if the mean LOS was used (the old situation) with the total overestimation and underestimation of the required ICU days if the prediction model was used (the new situation). The overestimation and underestimation in the old situation were calculated by subtracting the observed LOS from the mean LOS. The overestimation and underestimation in the new situation were calculated by subtracting the observed LOS from the predicted LOS. The mean LOS was used rather than the median LOS, because use of the median will favour the prediction model because the LOS is skewed.
Therefore, use of the mean LOS will result in a more conservative gain in comparison with the median LOS.

Both the old and new situations have three possible outcomes: negative, indicating that the ICU bed was reserved for too long and that the number of ICU days was overestimated; zero, indicating perfect prediction; or positive, indicating that the ICU bed was reserved for an insufficient period and that the number of ICU days was underestimated. The total overestimation and the underestimation were calculated for both the mean LOS approach and the LOS prediction model for both the old and the new situations.

\section{Results \\ Retrospective population}

The mean LOS was 8.76 days and the median LOS was 4.0 days (interquartile range 2.0 to 7.9 days). Overall, $6.8 \%$ of the patients were discharged from the ICU within 1 day after their surgical procedure, $37 \%$ within 3 days, $56 \%$ within 5 days and $69 \%$ within 7 days (Figure 1). Thirty-eight patients (7.3\%) were readmitted to the ICU after a stay shorter than 48 hours on the ward. Table 1 lists the retrieved data for variables that were thought to be potentially prognostic, broken down into patient characteristics, tumour and session characteristics, and postoperative complications within the first 72 postoperative hours. ICU mortality was $2.5 \%$ and total in-hospital mortality was $4.1 \%$.

\section{Univariate analysis}

The following preoperative variables (Table 1) were associated with longer LOS: older age $(P<0.001)$, American Society of Anesthesiology's Physical Status 3 or $4(P=0.001)$, presence of five out of seven comorbidities $(P<0.001$ to $0.14)$, squamous cell carcinoma $(P=0.003)$, transthoracic approach instead of transhiatal $(P<0.001)$, reconstruction using colon instead of stomach $(P=0.02)$, previous chemotherapy $(P=0.003)$ and lower forced expiratory volume in $1 \mathrm{~s}$ during preoperative screening $(P<0.001)$. Intraoperative variables associated with longer LOS were higher absolute amount of colloids administered $(P=0.01)$, greater absolute blood loss $(P=0.04)$, longer duration of vasopressor administration $(P=0.03)$, higher respiratory minute volume $(P=$ $0.005)$ and lower arterial oxygen saturation $(P<0.001)$. Patients with any complication occurring within 72 hours after surgery also had significantly longer LOS $(P<0.001)$.

\section{Preoperative, postoperative and intra-ICU multivariable models}

The multiple $r^{2}$ for the preoperative model was $21 \%$ and the optimism was $6 \%$; hence, the $r^{2}$ after validation was $15 \%$. The preoperative model had a $95 \%$ confidence interval $(\mathrm{Cl})$ with relative bounds between 0.5 and 2.5. This implies that LOS may be from $50 \%$ shorter to $254 \%$ longer than the mean LOS. Patient age $(P=0.001)$, presence of gastroesophageal reflux disease $(P<0.001)$, neurological comorbidity $(P<0.001)$ 
Critical Care Vol 11 No 2 Van Houdenhoven et al.

and a transthoracic instead of transhiatal approach $(P<$ 0.001 ) were the variables that contributed most to the increase in LOS for the preoperative model.

For the postoperative model, the multiple $r^{2}$ was $25 \%$ and the optimism was $9 \%$; the $r^{2}$ after validation was $17 \%$. The $95 \%$ $\mathrm{Cl}$ with relative bounds was comparable to that of the preoperative model. Apart from the variables included in the preoperative model, higher absolute amount of colloids administered $(P=0.03)$ and a maximum respiratory minute volume during the surgical procedure $(P<0.001)$ were the variables found to contribute to LOS in the postoperative model.

The multiple $r^{2}$ of the intra-ICU model was $56 \%$ and the optimism was $11 \%$, resulting in an $r^{2}$ of $45 \%$ after validation. The intra-ICU model had a $95 \% \mathrm{Cl}$ with relative bounds between 0.3 and 3.4 , implying that LOS may be from $70 \%$ shorter to $340 \%$ longer than the mean LOS. Complications occurring within 72 hours in the ICU (five complications had $P<0.001$ and two complications had $P<0.06$ ) were the variables found

Table 2

Multivariable preoperative, postoperative and intra-ICU linear LOS analyses

\begin{tabular}{|c|c|c|c|}
\hline & Preoperative model & Postoperative model & Intra-ICU model \\
\hline (Constant) & 1.26 & 0.44 & 1.82 \\
\hline Expected session time (min) & $1.10(1.01-1.21)$ & & \\
\hline Patient age (per decade) & $1.16(1.06-1.28)$ & $1.20(1.09-1.31)$ & $1.09(1.02-1.17)$ \\
\hline $\mathrm{FEV}_{1}(\mathrm{I})$ & $0.91(0.81-1.03)$ & $0.85(0.75-0.96)$ & \\
\hline Gastroesophageal reflux disease (yes/no) & $1.46(1.14-1.89)$ & $1.53(1.19-1.96)$ & \\
\hline Vascular comorbidity (yes/no) & $1.29(1.01-1.66)$ & $1.32(1.03-1.69)$ & \\
\hline Neurological comorbidity (yes/no) & $1.74(1.24-2.43)$ & $1.82(1.31-2.53)$ & \\
\hline Previous chemotherapy (yes/no) & $0.81(0.68-0.97)$ & & \\
\hline Previous radiotherapy (yes/no) & & & $0.78(0.63-0.97)$ \\
\hline Transthoracic approach (yes/no) & $2.13(1.74-2.62)$ & $1.79(1.44-2.24)$ & $1.21(1.05-1.40)$ \\
\hline Reconstruction using colon (yes/no) & $1.56(1.05-2.30)$ & $1.52(1.03-2.23)$ & \\
\hline Observed session time (min) & & $1.07(0.98-1.16)$ & \\
\hline Volume administration of colloids (liter) & & $1.14(1.01-1.29)$ & \\
\hline Absolute intraoperative blood loss (I) & & $0.94(0.87-1.02)$ & \\
\hline Absolute intraoperative urine production (I) & & $1.12(0.99-1.25)$ & \\
\hline Epidural analgesia administration (yes/no) & & $0.83(0.69-1.01)$ & \\
\hline Respiratory minute volume (I) & & $1.09(1.04-1.15)$ & $1.05(1.01-1.09)$ \\
\hline Positive end-expiratory pressure $\left(\mathrm{cmH}_{2} \mathrm{O}\right)$ & & $1.03(0.99-1.07)$ & \\
\hline Chylothorax surgical complication (yes/no) & & & $1.31(0.96-1.79)$ \\
\hline Anastomosis leakage complication (yes/no) & & & $1.83(1.47-2.28)$ \\
\hline Other complication (yes/no) & & & $1.71(1.38-2.10)$ \\
\hline Pulmonary nonsurgical complication (yes/no) & & & $1.97(1.72-2.26)$ \\
\hline Myocardial infarction (yes/no) & & & $1.54(0.93-2.56)$ \\
\hline Infection (yes/no) & & & $1.61(1.25-2.07)$ \\
\hline Other nonsurgical complication (yes/no) & & & $1.41(1.22-1.62)$ \\
\hline Multiple $\mathrm{r}^{2}$ & $21 \%$ & $25 \%$ & $56 \%$ \\
\hline Optimism & $6 \%$ & $9 \%$ & $11 \%$ \\
\hline Optimism corrected $\mathrm{r}^{2}$ & $15 \%$ & $17 \%$ & $45 \%$ \\
\hline
\end{tabular}

Unless stated otherwise, values are expressed as coefficient (95\% confidence interval). FEV1, forced expiratory volume in $1 \mathrm{~s}$; ICU, intensive care unit; LOS, length of stay. 

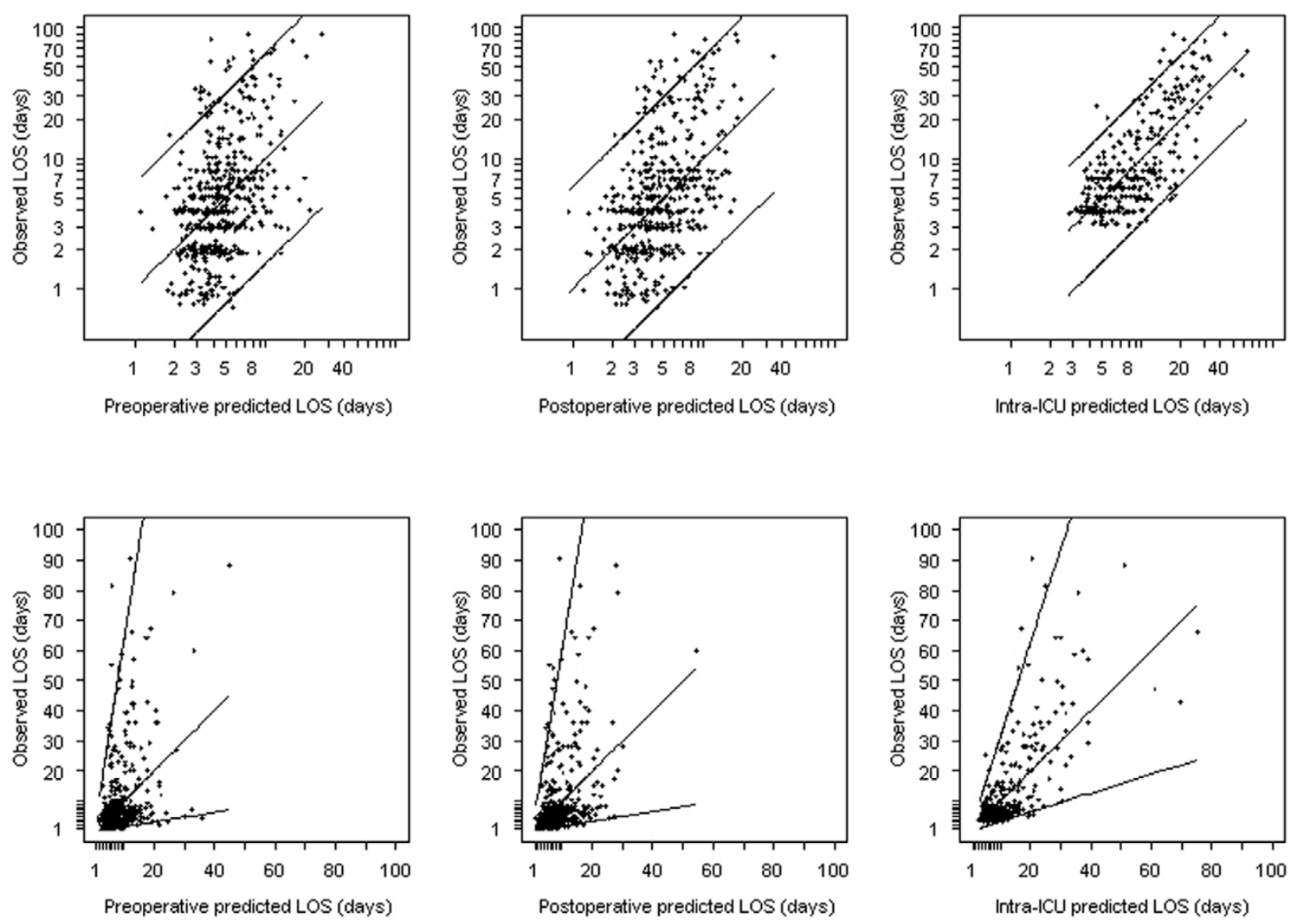

Calibration plots of the observed LOS against predicted LOS. These plots were constructed using the multivariable preoperative, postoperative and intra-ICU linear LOS models, on logarithmic scales (upper panel) and on untransformed scale with smearing factor (lower panels). ICU, intensive care unit; LOS, length of stay.

to contribute to LOS in the intra-ICU model. Results are shown in Table 2 and formulas to calculate the LOS of the preoperative, postoperative, and intra-ICU models can be found in Additional files 1, 2, and 3, respectively.

The goodness-of-fit of the three models is shown in Figure 2, which reveals considerable variation. Preoperative and postoperative LOS predictions exhibit variation and are not symmetrically distributed around the regression line. The LOS predictions of the intra-ICU model vary less, however, and are symmetrical. In addition, the prediction bounds of the intra-ICU model are much smaller than those of the preoperative and postoperative models.

\section{Model application}

Because the intra-ICU model has the highest $r^{2}$, this model was assessed for the second, prospective cohort of patients. This model included only patients who stayed in the ICU for at least three days. Of the 65 patients, 46 had a LOS longer than three days. The mean LOS for patients staying longer than three days in the ICU was 14.6 days, and so the 'remaining mean LOS' of 11.6 days (after subtraction of the first 3 ICU days) was used for comparison with the intra-ICU model. In the old situation (remaining mean LOS) and the new situation (prediction model), 10 out of 46 patients had an observed LOS longer than predicted (underestimation of ICU days), and the remaining 36 had an observed LOS shorter than predicted (overestimation of ICU days). In the old situation, these 10 patients together accounted for an underestimation of 220 ICU days; in the new situation they accounted for an underestimation of 155 ICU days. The other 36 patients together occupied the ICU for 213 days longer than predicted in the old situation, but in the new situation they occupied the ICU for 236 days longer than predicted (Table 3 ).

All in all, the total underestimation of ICU days decreased by 65 in favour of the prediction model; this is equal to $11 \%$ of the total ICU capacity of the study group. The total overestimation ICU days increased by 23 with the prediction model (in favour of prediction based on mean LOS). LOS was underes- 
Application of the model: underestimation and overestimation of old and new situation

\begin{tabular}{lccc}
\hline & & Situation & New \\
\cline { 2 - 4 } & Old & 10 \\
\hline Patients with underestimation $(n)$ & 10 & 155 & 36 \\
Total underestimated days & 220 & 36 & 236 \\
Patients with overestimation $(n)$ & 213 & 65 \\
Total overestimated days & - & 23 \\
Difference in underestimated days & - & &
\end{tabular}

In the old situation, estimation of LOS was based on the remeaning LOS after three days. The new situation used the prediction model. LOS, length of stay.

timated by the prediction model in 10 patients; this underestimation was less than in the old situation, however. Ultimately, 10 patient cancellations were prevented, which is equivalent to $15 \%$ of included patients.

\section{Discussion}

We showed that a predictive model incorporating characteristics of individual patients who underwent oesophagectomy for cancer enhanced the accuracy of estimated LOS. Key prognostic variables included patient's age, presence of gastroesophageal reflux disease, respiratory minute volume, transthoracic rather than transhiatal approach, and complications within the first 72 postoperative hours. We assessed three models and found that the intra-ICU model, which uses data from the first 72 hours in the ICU, had the best predictive performance. We found that use of this model in our clinical setting would have resulted in a gain of 65 ICU days over a 12month period. This is equivalent to $11 \%$ of the ICU capacity for this patient group. Moreover, $15 \%$ of cancellations of future surgical procedures could have been prevented.

Three types of related studies have been reported in the literature. First, we found reports of LOS prediction models that suggest specific therapeutic interventions in patient groups that may influence LOS $[22,23]$. Second, prediction models to determine risks for prolonged LOS have been developed [24$31]$. In these studies the investigators used preoperative, intraoperative and postoperative variables to fit a logistic model, with risk for prolonged LOS as the main outcome. This outcome measure is claimed to improve planning and therefore cost-effectiveness of hospitals. However, the results from these studies do not permit scheduling of individual patients on the ICU. They only calculate the risk for prolonged LOS given a certain cutoff point. The third type of study also uses individual patient characteristics to predict LOS, as in our study. However, they apply less sophisticated mathematical techniques (multiple linear regression), whereas the present study used logistic regression [32]. These models can only be used for medically homogeneous patient groups with a shorter and less variable LOS. In summary, the models proposed in these earlier studies are unsuitable for scheduling of individual patients in the ICU. In contrast, the prediction model proposed here does permit individual patient scheduling in the ICU on a day-to-day basis.

A typical example illustrates the value of the prediction model. A 79-year-old oesophagectomy patient without previous radiotherapy was operated on via a transhiatal approach; the measured maximum respiratory minute volume was 9.6 I during the surgical procedure, and various complications occurred within the first 72 hours in the ICU. The predicted LOS for that patient using our model is 84 days. This well exceeds the mean LOS of 8.76 days that was calculated using data in the hospital information system.

Prediction models, such as that proposed here, can improve quality of care and cost-effectiveness in an ICU, as was demonstrated in the prospective second cohort of patients we analyzed. Data required for the development and application of prediction models are typically available in every hospital. Therefore, prediction models can be used in almost any clinical setting, but they must be developed for specific groups of individual patients if the full benefit in terms of capacity gain is to be realized. The ICU typically occupies an important position in patient flow, and discharge of a patient typically allows new patients to enter the ICU. More accurate prediction of ICU discharge date therefore results in a more reliable and predictable care process, not just in the ICU but throughout the patient care pathway, including the operating room and the ward.

There are some limitations to our study. It was conducted among just one group at a single centre, which may limit the generalizability of our results to other centres. In addition, classification of variables will not be the same in all centres. The development of models like those proposed here requires effort. Also, some variables may change over time, and so the model should be updated periodically to maintain accuracy of 
prediction. Moreover, data such as pathological stage and how radical the surgical procedure is are typically only available during the second week postoperatively at our hospital, and so this information cannot be used as variables in a model during the first week. The extent of lymph node dissection was standardized in the surgical approach, and so an extra variable was not needed for this type of operation [33]. In the present study, the mean LOS at the ICU after oesophagectomy was long. The majority of patients had a LOS of more than 3 days. Estimates of ICU LOS in the literature vary, but the ICU LOS at our institution appears to be reasonable in comparison with those reports $[8,34,35]$. Clearly, ICU LOS prediction models are of greater value to hospitals with patient groups that have longer mean LOS. In our study, the particular patient group chosen was selected for that reason, so that we could experiment with the creation of such a model.

A strength of our study is that there is no selection bias; all patients were admitted to the ICU postoperatively according to protocol. Although our application sample differs statistically from the construction sample for some variables, the model appeared robust enough to make accurate predictions. Furthermore, a multiple imputation method was used to impute missing values, and so all patients were indeed included in the analysis.

\section{Conclusion}

We constructed, validated and tested three models, with incrementally enhanced precision, to predict LOS for individual patients in the ICU. The intra-ICU model proved able to predict LOS most accurately. For the highly variable LOS of oesophagectomy patients, this model appears to counter the commonly held view that LOS is unpredictable. Moreover, comparing the predictions of the model with historically determined mean LOS yielded significant improvement in terms of ICU capacity.

\section{Key messages}

- It is possible to construct a model to predict LOS in the ICU for an individual patient undergoing oesophagectomy with reconstruction for carcinoma, which has accuracy superior to that of prediction based on the mean LOS.

- These models can improve scheduling of patients in the ICU, yielding more efficient use of ICU beds and better quality of care as a result of fewer cancellations.

- Other models should be developed for other elective surgical procedures that require postoperative intensive care in order to improve efficiency and quality of care in the ICU.

\section{Competing interests}

The authors declare that they have no competing interests.

\section{Authors' contributions}

$\mathrm{MH}, \mathrm{DTN}, \mathrm{MJE}, \mathrm{GW}$ and GK made contributions to the concept, design and acquisition of data. DTN, MJE and EWS were responsible for the analysis and interpretation of data. HWT, DG, JB and GK were involved with clinical aspects of the study. All authors read and approved the final manuscript.

\section{Additional files}

The following Additional files are available online:

\section{Additional file 1}

A Word document showing calculation of the ICU LOS using the preoperative prediction model.

See http://www.biomedcentral.com/content/

supplementary/cc5730-S1.doc

\section{Additional file 2}

A Word document showing calculation of the ICU LOS using the postoperative prediction model.

See http://www.biomedcentral.com/content/ supplementary/cc5730-S2.doc

\section{Additional file 3}

A Word document showing calculation of the ICU LOS using the intra-ICU prediction model.

See http://www.biomedcentral.com/content/ supplementary/cc5730-S3.doc

\section{References}

1. Duke GJ: Metropolitan audit of appropriate referrals refused admission to intensive care. Anaesth Intensive Care 2004 32:702-706

2. Garrouste-Org, Montuclard L, Timsit JF, Reignier J, Desmettre T, Karoubi P: Predictors of intensive care unit refusal in French intensive care units: a multiple-center study. Crit Care Med 2005, 33:750-755.

3. Levin PD, Worner TM, Sviri S, Goodman SV, Weiss YG, Einav S, Weissman C, Sprung CL: Intensive care outflow limitation-frequency, etiology, and impact. J Crit Care 2003, 18:206-211.

4. Bakker J, Damen J, van Zanten AR, Hubben JH: Admission and discharge criteria for intensive care departments. Ned Tijdschr Geneeskd 2003, 147:110-115.

5. American Society of Anesthesiologists: New classification of physical status. Anesthesiology 1963, 24:111.

6. Rempe-Sorm V: Management of postoperative complications. In Barrett's Esophagus Edited by: Tilanus HW, Attwood SE. Dordrecht: Kluwer Academic Publishers; 2001:357-366.

7. Sobin $\mathrm{LH}$, Wittekind $\mathrm{CH}$, (editors): TNM Classification of Malignant Tumours (UICC) New Jersey: John Wiley \& Sons; 2002:60-65.

8. Avendano CE, Flume PA, Silvestri GA, King LB, Reed CE: Pulmonary complications after esophagectomy. Ann Thorac Surg 2002, 73:922-926.

9. Bartels $\mathrm{H}$, Stein $\mathrm{HJ}$, Siewert JR: Preoperative risk analysis and postoperative mortality of oesophagectomy for resectable oesophageal cancer. Br J Surg 1998, 85:840-844.

10. Ferguson MK, Martin TR, Reeder LB, Olak J: Mortality after esophagectomy: risk factor analysis. World J Surg 1997, 21:599-603. 
11. Law S, Wong KH, Kwok KF, Chu KM, Wong J: Predictive factors for postoperative pulmonary complications and mortality after esophagectomy for cancer. Ann Surg 2004, 240:791-800.

12. Tsutsui S, Moriguchi S, Morita M, Kuwano H, Matsuda H, Mori M, et al:: Multivariate analysis of postoperative complications after esophageal resection. Ann Thorac Surg 1992, 53:1052-1056.

13. Spiegelhalter DJ: Probabilistic prediction in patient management and clinical trials. Stat Med 1986, 5:421-433.

14. Steyerberg EW, Eijkemans MJ, Harrell FE Jr, Habbema JD: Prognostic modelling with logistic regression analysis: a comparison of selection and estimation methods in small data sets. Stat Med 2000, 19:1059-1079.

15. Steyerberg EW, Eijkemans MJ, Harrell FE Jr, Habbema JD: Prognostic modeling with logistic regression analysis: in search of a sensible strategy in small data sets. Med Decis Making 2001, 21:45-56.

16. Rubin DB, Schenker N: Multiple imputation in health-care databases: an overview and some applications. Stat Med 1991, 10:585-598.

17. Strum DP, May JH, Vargas LG: Modeling the uncertainty of surgical procedure times: comparison of log-normal and normal models. Anesthesiology 2000, 92:1160-1167.

18. Duan N: Smearing estimate: a nonparametric retransformation method. J Am Stat Assoc 1983, 383:605-610.

19. Efron B, Tibshirani RJ: An Introduction to the Bootstrap New York, NY: Chapman and Hall; 1993.

20. Steyerberg EW, Harrell FE, Borsboom GJJM, Eijkemans MJC, Vergouwe $Y$, Habbema JDF: Internal validation of predictive models: efficiency of some procedures for logistic regression analysis. J Clin Epidemiol 2001, 54:774-781.

21. Harrell FE, Lee KL, Mark DB: Multivariable prognostic models: Issues in developing models, evaluating assumptions and adequacy, and measuring and reducing errors. Stat Med 1996, 15:361-387

22. van de Pol MA, van Houdenhoven M, Hans EW, Boersma E, Bax JJ, Feringa HH, Schouten O, van Sambeek MR, Poldermans D: Influence of cardiac risk factors and medication on length of hospitalization in patients undergoing major vascular surgery. Am J Cardiol 2006, 97:1423-1426.

23. Van den BG, Wilmer A, Hermans G, Meersseman W, Wouters PJ, Milants I, et al.: Intensive insulin therapy in the medical ICU. $N$ Engl J Med 2006, 354:449-461.

24. Ammori BJ, Larvin M, McMahon MJ: Elective laparoscopic cholecystectomy: preoperative prediction of duration of surgery. Surg Endosc 2001, 15:297-300.

25. Collins TC, Daley J, Henderson WH, Khuri SF: Risk factors for prolonged length of stay after major elective surgery. Ann Surg 1999, 230:251-259.

26. Janssen DP, Noyez L, Wouters C, Brouwer RM: Preoperative prediction of prolonged stay in the intensive care unit for coronary bypass surgery. Eur J Cardiothorac Surg 2004, 25:203-207.

27. McMeekin DS, Gazzaniga C, Berman M, DiSaia P, Manetta A: Retrospective review of gynecologic oncology patients with therapy-induced neutropenic fever. Gynecol Oncol 1996, 62:247-253.

28. Rosenfeld R, Smith JM, Woods SE, Engel AM: Predictors and outcomes of extended intensive care unit length of stay in patients undergoing coronary artery bypass graft surgery. $J$ Card Surg 2006, 21:146-150.

29. Stoica SC, Sharples LD, Ahmed I, Roques F, Large SR, Nashef SA: Preoperative risk prediction and intraoperative events in cardiac surgery. Eur J Cardiothorac Surg 2002, 21:41-46.

30. Tu JV, Mazer CD, Levinton C, Armstrong PW, Naylor CD: A predictive index for length of stay in the intensive care unit following cardiac surgery. CMAJ 1994, 151:177-185.

31. Aronow HD, Peyser PA, Eagle KA, Bates ER, Werns SW, Russman PL, Crum MA, Harris K, Moscucci M: Predictors of length of stay after coronary stenting. Am Heart J 2001, 142:799-805.

32. Zimmerman JE, Kramer AA, McNair DS, Malila FM, Shaffer VL: intensive care unit length of stay: benchmarking based on Acute Physiology and Chronic Health Evaluation (APACHE) IV. Crit Care Med 2006, 34:2517-2529.

33. Lerut T, Coosemans W, Decker G, De Leyn P, Moons J: Surgical techniques. J Surg Oncol 2005, 92:218-229.
34. Chandrashekar MV, Irving M, Wayman J, Raimes SA, Linsley A: Immediate extubation and epidural analgesia allow safe management in a high-dependency unit after two-stage oesophagectomy. Results of eight years of experience in a specialized upper gastrointestinal unit in a district general hospital. BrJ Anaesth 2003, 90:474-479.

35. Kuo EY, Chang Y, Wright CD: Impact of hospital volume on clinical and economic outcomes for esophagectomy. Ann Thorac Surg 2001, 72:1118-1124. 